\title{
Etude du mécanisme des transferts d'eau et de soluté en zone non saturée avec prise en compte d'une fraction liquide immobile
}

\author{
PAR
}

\author{
J.-P. Gaudet, H. Jegat ex G. Vachaud \\ Institut de Mécanique, Laboratoire associé au CNRS. \\ Université Scientifique et Médicale de Grenoble
}

\section{Introduction}

L'étude des transferts couplés d'eau et de soluté en milieu non saturé recouvre une gamme de problèmes très variés et importants. On notera ainsi que la majorité des cas de pollution de nappe de surface résultent de la propagation d'un polluant déversé à la surface du sol et transitant à travers un sol à saturation variable. Les problèmes d'efficacité des engrais, ou de lessivage de sol présentent également un intérêt considérable en agronomie. Il y a donc lieu d'aborder cette étude avec soin pour bien comprendre les mécanismes mis en jeu et être ensuite en mesure d'agir avec un maximum d'efficacité.

Une intervention efficace doit notamment être basée sur la possibilité de prédiction correcte de l'évolution de la répartition spatio-temporelle de concentration du soluté en fonction des conditions imposées. L'outil privilégié pour répondre à ce but est le modèle numérique, à condition d'être certain de sa représentativité physique.

Nous avons voulu dans cette étude, mettre au point un modèle de transfert s'appuyant sur des essais de laboratoire réalisés dans des conditions relativement simples et bien contrôlées afin que les paramètres du modèle soient déterminés sans ambiguité. C'est pourquoi notre étude se limite au cas de l'écoulement unidirectionnel vertical, sans interaction soluté-matrice solide. $\mathrm{Ce}$ cas peut paraitre très restrictif, mais il est important de représenter correctement le mécanisme de transfert hydrodynamique avant de vouloir traiter le problème dans sa généralité, et notamment en prenant immédiatement en compte les phénomènes d'adsorption, d'exclusion ionique ou les réactions chimiques; ce qui ne pourra se faire à notre avis que dans un deuxième temps.
Même avec cette hypothèse restrictive, le problème reste complexe.

Nous nous proposons d'abord de présenter un schéma théorique relativement nouveau tenant compte du piégeage possible durant l'écoulement d'une partie du liquide présent dans le milieu poreux, et des échanges moléculaires entre cette fraction immobile et le fluide en mouvement (contenant un soluté). Nous passerons ensuite en revue les difficultés liées à l'obtention de résultats expérimentaux directement utilisables. Nous présenterons enfin certains essais réalisés récemment et utiliserons leurs résultats pour étudier les performances d'un modèle de simulation numérique, et la sensibilité des paramètres intervenant dans ce modèle.

\section{Equation des transferts}

Alors que l'étude de l'écoulement de fluides miscibles en milieux poreux saturés a fait l'objet d'un assez grand nombre de publications (notamment dans le domaine de l'intrusion d'eau salée dans les nappes aquifères : Bear, 1972 ; Fried-Combarnous, 1971), un nombre relativement limité d'articles a été consacré à l'étude du transfert de fluides miscibles dans un milieu à saturation variable. Ce type de problème a été abordé en physique du sol ou hydrologie (Biggar, Nielsen, 1967 ; Andersen-Sevel, 1974 ; Van Genuchten et al, 1974), en Génie chimique (Lapidus, Amundson, 1952 ; Villermaux et al, 1969) et en Génie pétrolier (Coats, Smith, 1964). On doit cependant noter que ces études ont été faites selon deux approches très différentes : dans un cas l'on a simplement repris le schéma classique de la dispersion hydrodynamique avec convection, utilisé lorsque le milieu est saturé, en ne faisant intervenir l'influence de la saturation partielle qu'au niveau de la vitesse du fluide ; dans l'autre cas au contraire l'on a supposé que la présence d'une phase gazeuse conduisait à la partition de la 
phase fluide entre une partie dite "immobile" et une autre dite "mobile". Nous examinerons ces deux approches successivement. Afin de pouvoir obtenir un système linéaire nous nous placerons dans le cas où le régime hydrodynamique est permanent. Clairement cette hypothèse suppose qu'en toute section du milieu poreux la vitesse du fluide est constante, seule change la concentration en soluté. Nous considérons enfin par la suite que le milieu poreux est inerte, que le fluide est de l'eau avec un soluté parfaitement miscible et que tous les transferts sont isothermes dans un milieu homogène.

La première approche, qui est la plus utilisée car elle découle de l'approche classique en milieu saturé, est celle que nous appelerons "dispersion convective" qui est représentée par l'équation :

$$
\frac{\partial C}{\partial t}=D_{a p} \frac{\partial^{2} C}{\partial z^{2}}-v_{0} \frac{\partial C}{\partial z}
$$

où : $C$ est la concentration massique en soluté dans la phase liquide $\left(\mathrm{ML}^{-3}\right)$

$D_{a p}$ est le coefficient de dispersion apparent $\left(\mathrm{L}^{2} \mathrm{~T}^{-1}\right)$

$v_{0}$ est la vitesse de pore, $v_{0}=q / \theta\left(\mathrm{LT}^{-1}\right)$

$q \quad$ est la vitesse de Darcy, dans ce cas constante $\left(\mathrm{LT}^{-1}\right)$

$\theta$ est la teneur en eau volumique $\left(\mathrm{L}^{3} / \mathrm{L}^{8}\right)$

$z \quad$ cst la cote (L)

$t \quad$ est le temps (T).

Cette approche suppose essentiellement que toute la solution liquide en place dans le sol participe à l'écoulement, la matrice solide n'intervenant que comme une frontière géométrique. Elle n'implique la détermination que d'un seul paramètre : le coefficient de dispersion apparent $D_{a p}$. On montrera par la suite que ce modèle se révèle incapable de reproduire curtaines courbes très dissymétriques d'évolution de la concentration en fonction du temps pour une section donnée (Jegat - 1975). C'est pourquoi l'on est amené à considérer la seconde approche où l'on suppose que la phase liquide est constituée de deux parties

- une fraction mobile qui éventuellement transporte un soluté ;

- une fraction immobile qui reste liée à la matrice solide.

Le soluté ne peut transiter entre les deux fractions que par diffusion moléculaire. La fraction immobile est constituée d'eau dans les pores en cul-de-sac et dans les agrégats, d'eau pelliculaire sur les grains solides, mais aussi d'eau piégée par la présence d'air dans les pores.

D'après la représentation schématique proposée ci-dessus, il est facile de concevoir que plus il y a d'air (plus la teneur en cau est faible), plus le volume d" "eau morte" est important, d'où l'intérêt d'étudier la validité de cette schématisation pour les transferts en sol non saturé.

En supposant toujours que le régime d'écoulement est établi (vitesse de Darcy constante) et en séparant la contribution relative à la fraction mobile et à la fraction immobile, l'équation (1) peut être écrite sous la forme :

$$
\frac{\theta_{i m}}{\theta_{m}} \cdot \frac{\partial C_{i m}}{\partial t}+\frac{\partial C_{m}}{\partial t}=D_{a p} \frac{\partial^{2} C_{m}}{\partial z^{2}}-v \frac{\partial C_{m}}{\partial z}
$$

où les indices $m$ et im réfèrent respectivement aux fractions mobile et immobile et où $v=q / \theta_{m}$ est la vitesse du fluide mobile.

On notera que l'écriture de l'équation (2) n'est valable que pour des vitesses d'écoulement suffisamment élevées, permettant de négliger la dispersion longitudinale dans la fraction immobile, c'est-à-dire le terme

$$
D_{i m} \frac{\theta_{i m}}{\theta_{m}} \frac{\partial^{2} C_{i m}}{\partial z^{2}}
$$

où $D_{i m}$ est le coefficient de diffusion dans le fluide immobile

On suppose de plus qu'entre les deux fractions se produit un échange défini par une dynamique correspondant à l'équation (Coats - Smith, 1964):

$$
\theta_{i m} \frac{\partial C_{i m}}{\partial t}=\alpha\left(C_{m}-C_{i m}\right)
$$

où $\alpha$ est le coefficient de transfert $\left(\mathrm{T}^{-1}\right)$ à travers la surface de contact inconnue entre fractions mobile et immobile.

On notera que le schéma avec partition de la phase fluide, (équations 2-3), se rapproche du schéma de dispersion convective dans les cas suivants:

- coefficient de transfert très grand (échange quasi instantané)

et/ou

- teneur en eau mobile voisine de la teneur en eau totale.

Le schéma avec partition étant cependant plus complet. puisque l'autre en est finalement un cas particulier, sera adopté pour la suite de cette étude. Notons qu'il implique la détermination de trois paramètres : le coefficient de dispersion apparente $D_{a p}$, le coefficient de transfert $\alpha$ et le coefficient de partition, ou la fraction mobile $f=\theta_{m} / \theta$.

Ces paramètres doivent être déterminés à partir d'essais effectués dans des conditions parfaitement connues, et contrôlées, done de préférence des essais de laboratoire. Le coefficient de dispersion $D_{a p}$ a fait l'objet de nombreux travaux (Pfankuch, 1962 ; Klotz et Moser, 1974), et c'est le paramètre pour lequel on dispose du plus grand nombre de clonnées, bien que la majorité des travaux antérieurs ne soient relatifs qu'à des sols totalement saturés. A notre connaissance, les deux autres paramètres $f$ et $\alpha$ n'ont été calculés que deux fois à partir des concentrations de l'effluent : - par Coats et Smith (1964), pour des sols totalement saturés $\left(\theta_{m}\right.$ très proche de $\left.\theta\right)$, et à partir d'une condition à la limite inférieure correspondant à une colonne semiinfinie;

- par Van Genuchten (1974) en présence d'adsorption et à partir de la même condition à la limite inférieure.

Afin de définir entièrement le problème, et d'en obtenir des solutions, il importe enfin de décrire les conditions initiales et aux limites. Pour les exemples que nous traiterons par la suite, ces conditions, en terme de concentration, sont les suivantes:

* La condition initiale est :

$$
t<0 \quad C_{m}(z, t)=0, \quad C_{i m}(z, t)=0
$$

ce qui suppose qu'initialement le sol est sujet à un écoulement d'eau pure en régime permanent.

* La condition à la limite supérieure traduit la conservation de flux de sel à travers la surface supérieure $(z=0)$ de la colonne, soit :

$$
q C_{o}=\lim _{z \rightarrow 0^{+}}\left(q C_{m}-D_{a p} \theta_{m} \frac{\partial C_{m}}{\partial z}\right)
$$

où $C_{o}$ est la concentration de la solution infiltrée.

Dans les cas que nous présenterons, la concentration $C_{o}$ vaut :

$$
\begin{array}{ll}
\text { - pour } t<0 & : C_{o}=0 \\
\text { - pour } t \leqslant t<t_{1}: C_{o}=\text { constante } \\
\text { - pour } t \geqslant t_{1} & : C_{o}=0
\end{array}
$$

Ceci correspond soit à une condition de créneau de concentration pour $z=C$, soit à une condition d'échelon lorsque $t_{1}$ devient infini 
* Pour la limite inférieure enfin, nous n'écrirons pas la condition de colonne semi-infinie qui est la plus utilisée, mais une condition plus physique qui consiste à considerer que la dispersion (très proche de la diffusion moléculaire) dans le tube à la sortie de la colonne est négligeable devant la dispersion apparente à l'intérieur de la colonne, soit :

$$
\lim _{z \rightarrow L^{+}}\left(\frac{\partial C_{m}}{\partial z}\right)=0
$$

On notera que ces conditions ne sont pas limitatives. On peut notamment considérer d'autres conditions initiales, à condition de connaitre la répartition spatiale des concentrations à l'instant $t=0$

\section{Analyse de la représentativité des mesures}

Avant de vouloir analyser les résultats d'essais, ou extrapoler les données obtenues en laboratoire à un cas de terrain, il y a lieu de se montrer extrêmement critique sur de nombreux points.

En premier lieu les conditions d'expérience doivent être soigneusement définies. Il est évident que la reconstitution en laboratoire d'un échantillon de sol (généralement remanié) dans une colonne ne peut pas représenter la réalité du terrain. Les essais sur ce type d'échantillon devront essentiellement servir à déterminer les paramètres caractéristiques des transferts, et mettre au point un modèle de simulation qui pourra être ensuite utilisé comme outil objectif de prévision sur le terrain, à condition de lui injecter des paramètres représensentatifs. On notera en outre pour que les essais sur colonne soient utilisables, il faudra veiller à éliminer tous les effets parasites, tels que les écoulements préférentiels le long des parois qui interviennent généralement lors d'écoulements en charge, mais ne peuvent pas exister si la pression effective de l'eau est négative (transferts en milieu non saturé).

Les conditions initiales et aux limites doivent être également particulièrement bien définies. Pour obtenir une répétitivité satisfaisante des essais, il y a lieu d'avoir des colonnes suffisamment longues (Klotz-Moser, 1974). De plus les volumes morts dus aux circuits d'alimentation, tant à l'entrée qu'à la sortie des colonnes, doivent être très faibles devant le volume d'eau contenue dans le milieu poreux afin que la perturbation introduite par ces volumes soit négligeable.

Mais le point le plus crucial est finalement la signification physique des mesures, et leur validité. L'information la plus généralement utilisée dans les essais de laboratoire consistant au déplacement d'un fluide présent dans une colonne de sol par un autre miscible avec le premier est la courbe de sortie, ou courbe représentant la variation en fonction du temps de la concentration dans l'effluent de la colonne. Des techniques de convolution sont alors appliquées entre la courbe de sortie et la condition de créneau ou d'échelon imposée à l'entrée pour obtenir la "fonction de transfert" représentative de l'essai.

Si l'on admet cependant la validité physique du schéma de fractionnement entre fluide mobile et immobile, la concentration de l'effluent correspond seulement à celle de la fraction mobile (soit $C_{m}$ d'après nos notations) et non pas à la concentration $C$ de la solution présente dans le milieu poreux à la sortie de la colonne. Cette dernière valeur est donnée par l'identité :

$$
C . \theta=C_{m} \cdot \theta_{m}+C_{i n} \cdot \theta_{i m}
$$

avec :

$$
\theta=\theta_{m}+\theta_{i m}
$$

Quand la fraction d'eau immobile est importante, la forme des courbes de sortie pour une colonne suffisamment longue est assez caractéristique (très fortement assymétrique) pour que l'on ne puisse pas ajuster de manière satisfaisante un schéma de dispersion-convective classique (Jegat … 1975). Par contre lorsque la fraction immobile est très faible, ce schéma peut reproduire de manière satisfaisante les courbes expérimentales. Cependant, le coefficient $D_{a p}$ ainsi déterminé ne sera représentatif que de l'essai sur lequel l'ajustement a été fait et ne pourra en aucun cas être étendu à d'autres conditions.

De plus, les courbes de sortie intègrent tous les phénomènes pouvant intervenir lors du transit du soluté dans le massif poreux. En particulier on peut être en présence d'un écoulement quasi-stable (Dagan, 1966), c'est-à-dire que les contrastes de densité ou de viscosité font apparaitre des instabilités pendant un temps limité, ces instabilités disparaissent ensuite. Le temps de développement maximum de ces instabilités, $t_{m a x}$, peut étre très court par rapport au temps de transit du soluté dans la colonne, dans ce cas l'analyse n'est pas affectée par ce phénomène. Par contre $t_{\max }$ peut être relativement grand tout en restant inférieur au temps de transit ; dans ce cas les courbes de sortie ne permettent pas de s'en apercevoir (Jegat, 1975), et l'on attribuera à la dispersion ou à un échange quelconque un mélange provenant d'instabilités hydrodynamiques. Ces courbes de sortie peuvent enfïn aussi intégrer éventuellement les effets des hétérogénéités locales ou du mélange créés par la récupération de l'effluent à l'extrémité des colonnes.

Pour ces diverses raisons, nous pensons qu'il est indispensable de compléter l'information "courbe de sortie" par des mesures de concentrations faites dans le milieu poreux luimême en différentes sections et qui, associées à une mesure locale des teneurs en eau, permettent d'accéder à une analyse bien supérieure du phénomène observé. Mais là encore il s'agit d'être prudent car les mesures effectuées pourront correspondre soit essentiellement à la concentration $C_{m}$ de la partie mobile (si l'on effectue par exemple des prélèvements de solution à partir de bougies poreuses), soit à la concentration totale $C$ dans la section de contrôle (si l'on détermine l'activité d'un traceur isotopique radioactif par comptage direct).

La méthode que nous avons nous-même développée et utilisée est une méthode de mesure non destructive de la concentration totale $C$ basée sur une mesure locale de la résistivité de la solution (ce qui nous a conduit à utiliser une solution ionique). Cette technique a été décrite en détail par ailleurs (Gaudet, 1974) ; brièvement elle repose sur la mesure simultanée dans une section de la quantité d'ions présents dans un volume représentatif de milieu poreux (sonde résistive annulaire) et de la teneur en eau par absorption de rayonnement gamma. En couplant les deux mesures, on obtient directement la concentration massique $C(g / 1)$ totale de la solution à la cote $z$ et au temps $t$

On peut conclure partiellement à ce niveau en disant qu'il faut analyser avec beaucoup de soin les résultats expérimentaux relatifs à la dispersion, car on prend le risque d'ajuster (plus ou moins bien) un modèle mathématique à ces résultats, avec des paramètres qui n'ont pas la signification physique qu'on veut bien leur donner. Cette analyse une fois faite, le schéma mathématique décrivant le phénomène observé doit naturellement être basé sur des hypothèses en parfait accord avec les conditions expérimentales. 


\section{Résultats et discussion}

Notre but étant de mettre au point un modèle numérique permettant de simuler les transferts d'eau et de soluté dans la zone non saturée, partant des relations (2), (3), (4), (5), (6) et (7) qui représentent un certain schéma physique, nous devons d'abord contrôler la précision de la discrétisation (notamment l'influence du bruit numérique), ce qui a été fait en utilisant certaines solutions analytiques comme test; puis étudier l'aptitude du modèle à simuler des essais réels; et enfin nous intéresser à la sensibilité du modèle à toute variation des paramètres physiques intervenant dans les équations de base.

\section{a) Discrétisation}

Le schéma de discrétisation adopté pour la simulation est décrit en détail par Jegat (1975).

Très brièvement la résolution numérique est faite par la méthode des différences finies, selon un schéma explicite. Le terme de convection $(v \partial C / \partial z)$ est exprimé en différence arrière, ce qui donne des critères de stabilité moins sévères que pour une écriture en différence centrale, à condition de prendre en compte la diffusion numérique due à l'approximation au premier ordre :

- sur la dérivée par rapport au temps (schéma explicite) - sur le terme de convection (différence arrière).

Ceci revient à retrancher un terme de diffusion numérique $D N$ au coefficient de dispersion apparent $D_{a p}$, (Chaudhari, 1971), soit :

$$
\begin{gathered}
D A=D_{a p}-D N \\
\text { avec : } \quad D N=1 / 2 v(\Delta z-v \Delta t)
\end{gathered}
$$

où $\Delta z$ et $\Delta t$ sont respectivement les pas d'espace et de temps. Grâce à la maitrise de la stabilité et de la diffusion numé rique, cette discrétisation permet l'utilisation de mini-ordinateurs de bureau par le faible nombre de mémoires nécessaires (résolution explicite), tout en utilisant des pas de temps relativement grands. Tous nos claculs ont ainsi été effectué sur un ordinateur de bureau Hewlett-Packard modèle 10.

\section{b) Vérification du schéma numérique}

Deux types de vérifications ont été effectuées: d'une part sur la précision absolue, d'autre part sur la validité de la relation (7) fixant une condition à la limite inférieure de colonne de longueur finie. Pour ce faire on a utilisé deux solutions analytiques comme test de contrôle.

Dans un premier temps, et nous plaçant dans le cas d'une colonne semi-infinie ( $C \rightarrow 0$ lorsque $z \rightarrow \infty$ ), nous avons comparé la solution numérique avec une solution analytique donnée par Coats et al. (1964) pour la concentration dans la phase mobile uniquement. La comparaison a été faite pour deux vitesses très différentes, l'une très élevée pour des écoulements naturels d'eau en milieu poreux ( $q=207 \mathrm{~cm} / \mathrm{h}$ ), l'autre plus courante $(q=9,36 \mathrm{~cm} / \mathrm{h})$. Dans le premier cas, la convection est prépondérante, alors que dans le second cas, la dispersion devient plus importante. Les valeurs comparées des solutions numériques et analytiques sont présentées figure 1 ; elles sont dans les deux cas identiques.

Si l'on inclus toutefois dans le schéma numérique la relation (7) correspondant à une longueur de colonne finie, la simili-
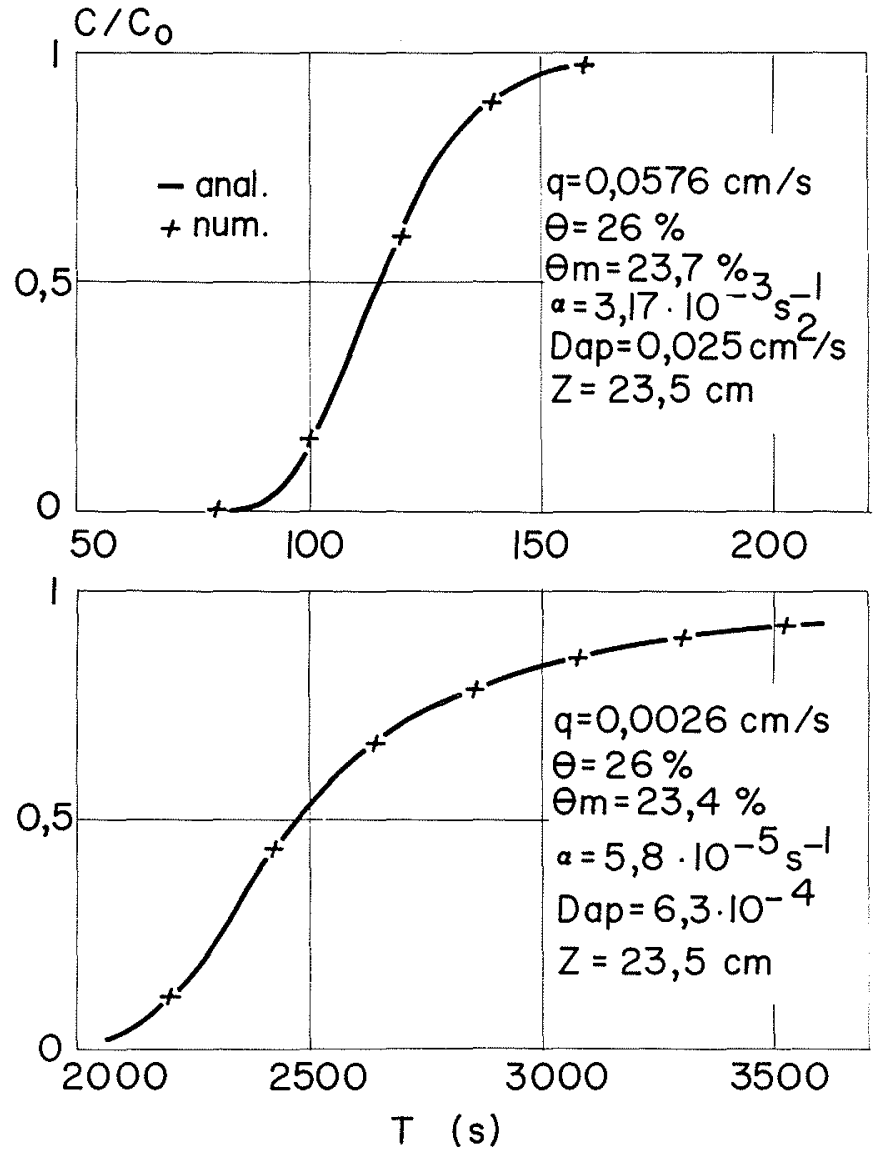

$1 /$ Comparaison entre solutions analy tiques (ligne continue) et numériques (croix) obtenues dans une colonne semi-infinie à la cote $z=23.5 \mathrm{~cm}$ pour un échelon de concentration à l'entrée et deux vitesses d'écoulement.

tude n'existe plus avec la solution analytique de Coats correspondant à une colonne semi-infinie (figure 2). Il est remarquable de noter que les deux solutions ne convergent pas vers une valeur unique, même quant la longueur de la colonne augmente, ceci étant encore vrai pour une colonne plus longue ( $94 \mathrm{~cm}$, cas non présenté ici).

Cette solution numérique pour une colonne de longueur finie a été également testée sur une solution analytique due à

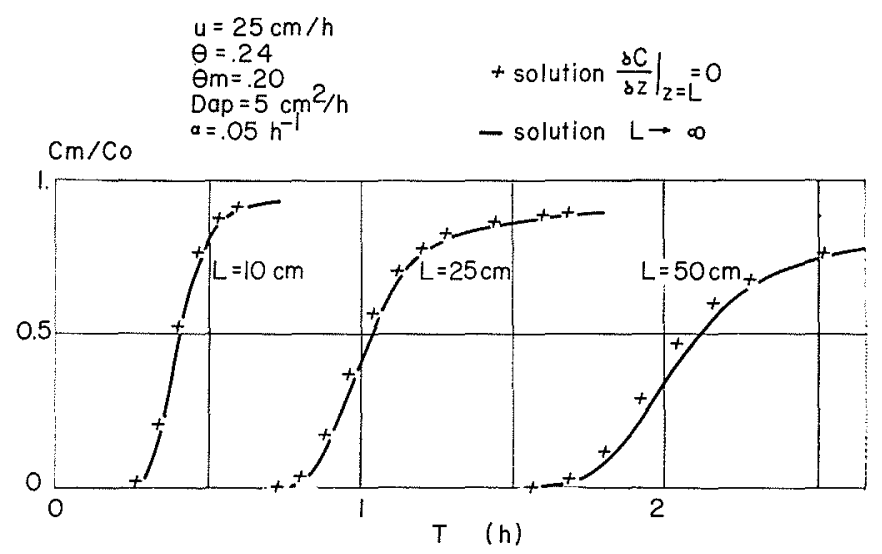

$2 /$ Comparaison entre solutions analytiques (ligne continue) pour une colonne semi-infinie, et solutions numériques (croix) obtenues à la sortie d'une colonne de longueur $L$ pour un échelon de concentration à l'entrée et différentes valeurs de $L$. 
Brenner (1962), qui résoud le système d'équations (1), (4), (5), (6), et (7), c'est-à-dire pour un transfert de soluté sans phase immobile et elle s'avère exacte. La discordance notée dans le paragraphe précédent est donc bien due à l'écriture de la condition à la limite inférieure. Cette constatation est importante, car elle montre le danger à vouloir faire correspondre une courbe de sortie obtenue sur colonne de longueur finie à la solution d'un modèle mathématique qui considère généralement une colonne semi-infinie. La similitude entre solution analytique correspondant à une colonne semi-infinie et les mesures n'existe que pour des sections de contrôle à l'intérieur de la colonne, suffisamment loin de l'extrémité inférieure pour ne pas ressentir l'effet de bout, et pas trop près de l'entrée pour que le régime de dispersion soit établi (Klotz et Moser, 1974). Notons encore que la solution due à Coats et Smith ne donne que la concentration dans la phase mobile, mais que Van Genuchten (1974), propose une solution analytique donnant à la fois la concentration dans les deux phases pour une colonne semi-infinie.

\section{c) Simulation de cas réel}

On considére deux exemples correspondant à la propagation dans une colonne de sol uniforme d'une variation de concentration saline lors d'un écoulement à débit constant soit dans le cas d'une condition d'échelon, soit dans le cas d'une condition de créneau imposée à l'entrée de la colonne.

Des essais ont été effectués en laboratoire sur une colonne verticale de sable fin très homogène, mesurant $93,5 \mathrm{~cm}$ de haut, $60 . \mathrm{mm}$ de diamètre intérieur. Des sondes de mesure résistives étaient implantées aux cotes $z=7,22,37,67$ et $82 \mathrm{~cm}$, un système de scrutation automatique permettant de mesurer la concentration en chaque section toutes les minutes. Les teneurs en eau étaient contrôlées chaque $5 \mathrm{~cm}$ grâce à une plateforme de mesure comportant une source d'Americium 241 de $100 \mathrm{mCi}$ et un détecteur à photomultiplicateur se déplaçant dans le plan vertical (Vachaud - Thony, 1970). La condition d'écoulement permanent à débit constant était imposée par l'intermédiaire d'une pompe volumétrique à double corps permettant de passer instantanément d'un débit $Q$ d'eau pure à un débit $Q$ d'eau salée $\left(\mathrm{CaCl}_{2}\right)$ de concentration donnée. Enfin la concentration de l'effluent était mesurée par un écoulement dans une sonde de platine scrutée également toutes les minutes.

Les conditions imposées pour les deux essais simulés ici étaient les suivantes:

- vitesse de Darcy : $10,8 \mathrm{~cm} / \mathrm{h}$ correspondant à une teneur en eau moyenne de $0.26 \mathrm{~cm}^{3} / \mathrm{cm}^{3}$ (porosité $0.37)$

- concentration de la solution saline : $3 \mathrm{~g} /$ litre de $\mathrm{CaCl}_{2}$.

Les résultats expérimentaux correspondant au premier essai (condition d'échelon à l'entrée) sont représentés fig. 3a et $3 \mathrm{~b}$ sous forme de variation de concentration relative dans les différentes sections de contrôle et à la sortie en fonction du temps. Pour vérifier lequel des deux schémas présentés au début de cet article était le plus valable, on a considéré successivement un modèle de simulation numérique basé sur l'un et l'autre cas.

On a d'abord résolu un modèle correspondant au schéma de la "dispersion convective", soit à la solution du système d'équations (1), (4), (5), (6) et (7). Les résultats calculés sont présentés sur la figure $3 \mathrm{a}$; le coefficient $D_{a p}$ étant déterminé par ajustement "à l'oeil" sur la section $Z=22 \mathrm{~cm}$. La concordance n'est pas excellente et surtout il n'est pas possible de reproduire les "queues" observées sur les courbes expérimentales, pour les fortes valeurs de $C / C_{o}(>0.75)$.

$$
q=10.8 \mathrm{~cm} / \mathrm{h}-\theta=0.257 \mathrm{~cm}^{3} / \mathrm{cm}^{3}
$$

a)

$$
\text { Dispersion convective } \quad D_{a p}=7 \mathrm{~cm}^{2} / \mathrm{h}
$$

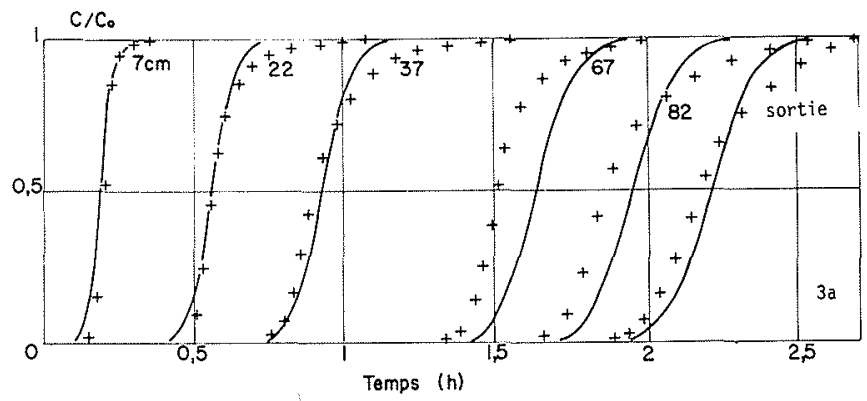

+ valeurs expérimentales

- simulation numérique

b)

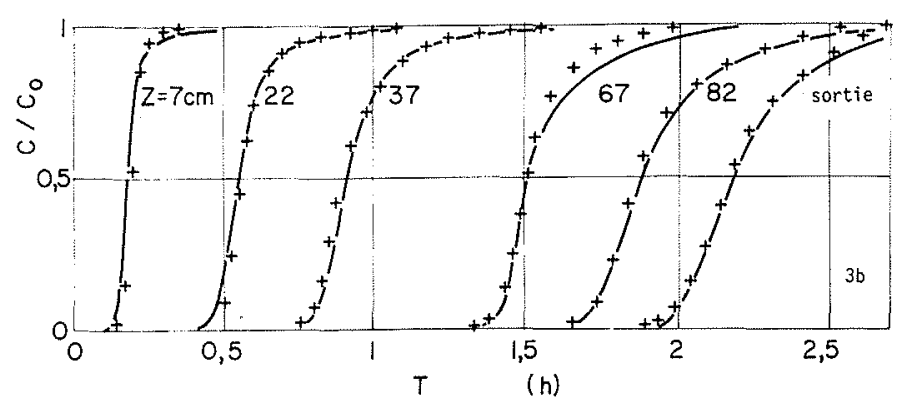

Dispersion et fraction immobile $D_{\text {ap }}=6 \mathrm{~cm}^{2} / \mathrm{h}, \alpha=0.05 \mathrm{~h}^{-1}, f=0.96$

$3 /$ Variation de concentration relative dans la colonne et à la sortie à la suite d'un échelon de concentration à l'entrée.

a/ Comparaison entre valeurs expérimentales (points) et courbes simulées (trait plein) avec le schéma de "dispersion convective"

b/Comparaison entre valeurs expérimentales (points) et courbes simulées (trait plein) en tenant compte d'une fraction immobile correspondant à une teneur en eau de $0.01 \mathrm{~cm}^{3} / \mathrm{cm}^{3}$.

Ce même essai est ensuite simulé en considérant qu'une partie du liquide est liée à la matrice solide, ce qui revient à résoudre le système des équations (2), (3), (4), (5), (6) et (7). Les valeurs moyennes des paramètres ajustés à l'oeil sur la section $Z=22 \mathrm{~cm}$ sont données en légende de la figure $3 . \mathrm{b}$ où sont reportées en traits pleins les courbes obtenues par solution numérique. On notera que dans ce cas la valeur $C / C_{0}$ pour les sections $7,22,37,67$ et $82 \mathrm{~cm}$ représente la concentration totale pondérée (relation 8 ) qui est celle que l'on mesure, alors que la valeur $C / C_{0}$ pour la courbe de sortie est représentative de la phase mobile uniquement. La concordance entre le calcul et l'expérience est cette fois très satisfaisante, sauf pour la section $z=67 \mathrm{~cm}$, où du fait d'une faible hétérogénéité la teneur en eau est localement plus faible pour cet essai, ce dont on n'a pas tenu compte pour le calcul.

Les résultats relatifs au second essai sont reportés fig. 4 . Dans ce cas la durée du créneau vaut $t_{1}=0.33 \mathrm{~h}$ (relation 6). Cet essai a été simulé uniquement en utilisant les valeurs des paramètres obtenues par ajustement dans l'essai précédent réalisé, rappelons le, avec la même vitesse moyenne et la même valeur de concentration de la solution saline. Ceci met en valeur la répétitivité des phénomènes physiques mis en jeu, tant expérimentalement que pour le calcul à partir du modèle pro- 


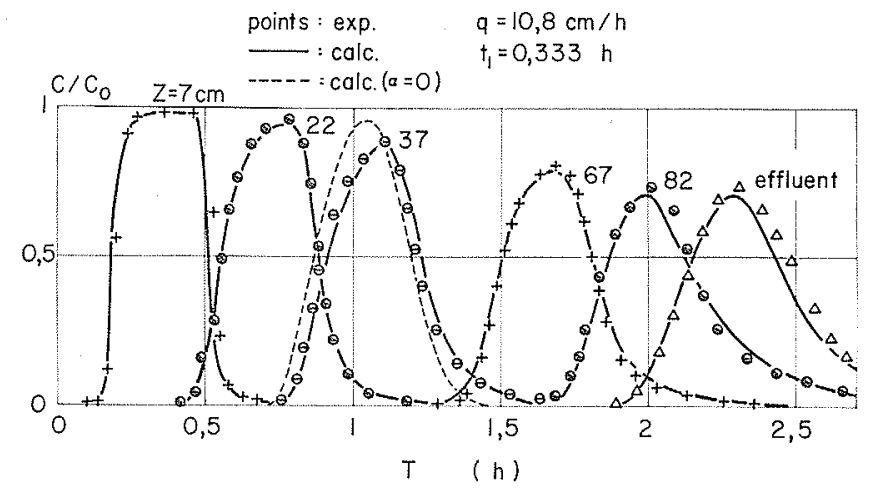

$4 /$ Variation de concentration relative dans la colonne et à la sortie à la suite d'un créneau de concentration à l'entrée pour un essai effectué à la même vitesse que dans le cas fig. 3 .

Comparaison entre mesures (points) et courbes simulées (trait plein) en tenant compte d'une fraction immobile, et utilisant

les paramètres obtenus (figure $3-b$ ). La courbe en pointillée $(z=37)$ serait obtenue par le schéma dispersion-convective.

posé. Les courbes reportées en traits pleins, fig. 4, correspondent à la solution numérique du schéma avec partition. Si l'on prend le schéma dispersion-convective simple, on obtient, à titre d'exemple, la courbe en traits discontinus pour la section $Z=37 \mathrm{~cm}$.

Ces résultats mettent clairement en évidence la supériorité du schéma avec partition, qui représente sûrement mieux la réalité physique que le schéma classique.

\section{d) Sensibilité des paramètres}

Il reste enfin, pour que cette présentation soit complète, à tester la sensibilité du modèle présenté à toute variation de grandeur des paramètres. Ce test a un double but : d'une part justifier à posteriori les méthodes d'identification des paramètres utilisés précédemment, d'autre part définir l'influence des variations dues aux hétérogénéités naturelles sur les résultats numériques. On s'est place dans le cas d'une condition d'échelon imposé à $z=0, t=0$ (qui correspond à l'exemple le plus souvent traité dans la littérature), et partant des valeurs numériques suivantes:

$$
\begin{aligned}
& D_{a p}=5 \mathrm{~cm}^{2} / \mathrm{h}, \alpha=0.05 \mathrm{~h}-1, \\
& \theta=0.24 \mathrm{~cm}^{3} / \mathrm{cm}^{3} \text { et } f=0.833
\end{aligned}
$$

obtenues lors d'essais analogues à ceux que nous avons présentés ici, mais avec un flux de $5 \mathrm{~cm} / \mathrm{h}$, on a étudié numériquement l'évolution de la courbe de concentration relative à la cote $z=50 \mathrm{~cm}$ lorsqu'un des trois parametres caractéristiques $\left(\alpha, f\right.$ et $\left.D_{a p}\right)$ varié, les deux autres étant laissés fixes. Les résultats sont reportés fig. 5,6 et 7 .

On notera que $f$ est un paramètre très important, car il agit à la fois sur le temps auquel apparait le soluté et sur la longueur des queues de courbe. Le parametre $\alpha$ agit essentiellement sur la pente de la courbe de transition et sur celle de la queue de courbe. Le paramètre $D_{a p}$ semble etre le moins influent et n'agit que partiellement sur la pente de la partie initiale de la courbe.

On pourra cependant noter que pour de fortes valeurs de $f$ (proches de 1 ), le coefficient $D_{a p}$ devient le plus important, ce qui est prévisible puisqu'on se rapproche du cas décrit par l'équation (1), où seul le paramètre $D_{a p}$ intervient. Mais bien que dans notre exemple $f$ soit voisin de 1 , le schéma avec partition utilisé permet de reproduire des queues importantes, ce qui est impossible à partir de la résolution de l'équation (1).

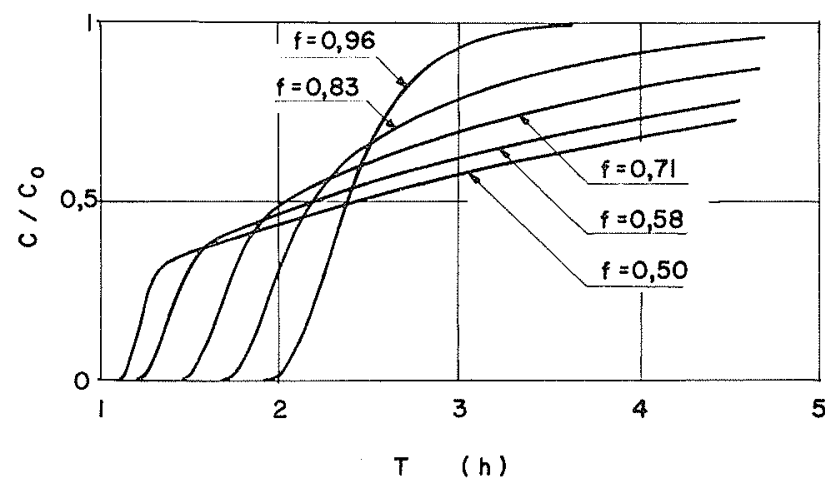

$5 /$ Influence de la fraction mobile $f$

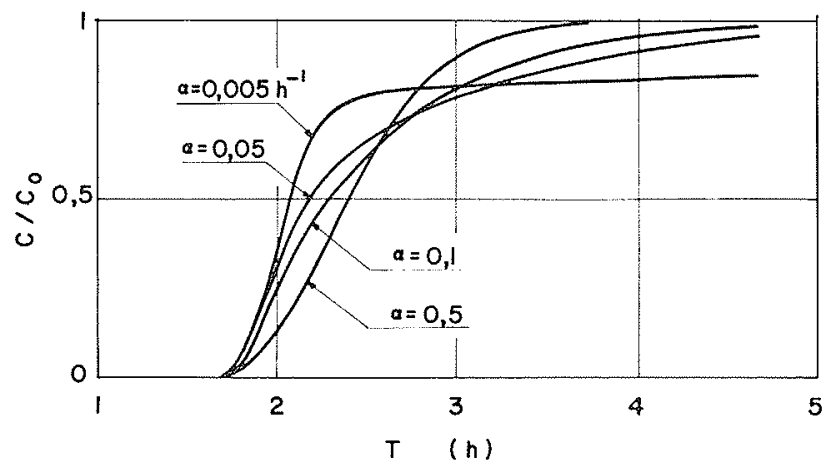

$6 /$ Influence du coefficient de transfert $\alpha$

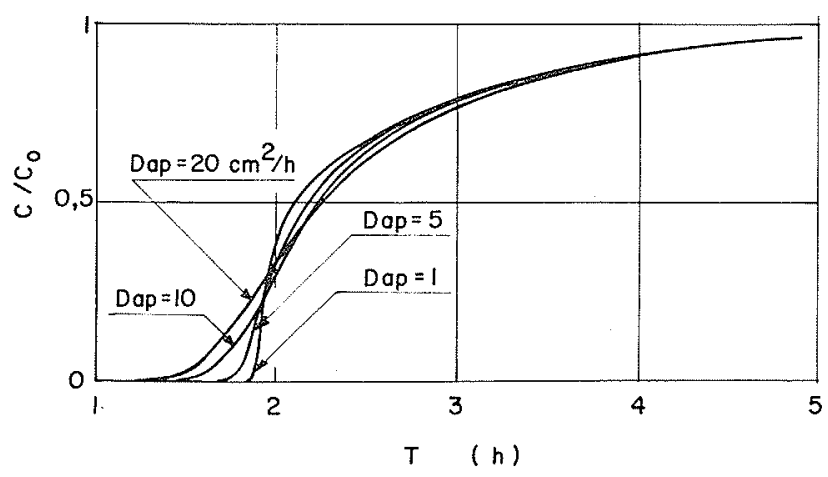

$7 /$ Influence de la dispersion apparente $D_{a p}$.

5/6/7/ Etude de la sensibilité des paramètres. Calcul de l'influence d'un des paramètres sur la courbe de variation de concentration obtenue à $z=50 \mathrm{~cm}$ pour un échelon de concentration à l'entrée :

Il ressort de cette étude de sensibilité que la méthode d'identification "au jugé" est bonne puisque chaque paramètre influe différemment sur l'allure des courbes de concentration relative. La variation de $f$ avec la texture du milieu risque cependant de poser des problèmes pratiques importants.

\section{Conclusion}

Nous venons de montrer que le schéma de dispersion convective dans un sable partiellement saturé n'est pas suffisamment représentatif. La prise en compte de deux fractions d'eau, l'une mobile et éventuellement vecteur d'un soluté, l'autre immobile jouant le rôle de source ou puits permet de simuler avec une très bonne précision les variations de concen- 
tration dans une colonne de sable inerte. La résolution numérique par un schéma de différences finies explicites permet de prendre en compte des conditions aux limites plus proches de la réalité physique que les solutions analytiques supposant les colonnes semi-infinies, et permet de se donner une loi $C_{0}(t)$ quelconque pour la concentration imposée à la frontière supérieure. Enfin un tel schéma explicite, dont on contrôle la stabilité et qui permet d'utiliser des pas de temps assez grands compatibles avec une bonne précision, peut être résolu sur des mini-ordinateurs.

Notre traitement du problème ne prétend pas être exhaustif, mais nous espérons avoir montré qu'il est nécessaire d'apporter beaucoup de soin à l'analyse des données, et à la validité des hypothèses formulées.

Pour des études futures il est nécessaire d'explorer dans toute la gamme des teneurs en eau la variation des paramètres $\alpha, f$ et $D_{a p}$, en fonction de la teneur en eau et de la vitesse. Ceci devrait conduire à la simulation, (donc la prédiction), d'un écoulement couplé d'eau et de soluté en régime non permanent dans la zone non saturée, cas le plus généralement rencontré dans la nature. Il sera enfin nécessaire d'envisager rapidement le cas des interactions entre soluté et matrice poreuse (adsorption, exclusions ioniques, ou changement de phases).

Nous estimons néanmoins qu'une étape intermédiaire indispensable est la mise au point d'un schéma simple et fiable du phénomène le plus important qui est celui du transport hydrodynamique. C'est tout le sens de cette intervention.

Cette étude a été faite dans le cadre d'une convention avec le Haut Comité pour l'Environnement (Convention 73/76). Elle a donné lieu à une thèse de Docteur-Ingénieur (H. Jegat -.. "Ecoulement de fluides miscibles en milieux partiellement saturés - Expérience et simulation" Université Scientifique et Médicale de Grenoble - Décembre 1975).

Nous tenons à remercier le Professeur P. Wierenga, New-Mexico State University, Las Cruces (New-Mexico - USA), pour l'aide qu'il nous a apportée dans l'analyse des résultats et le traitement des données durant son séjour sabbatique à Grenoble.

\section{Bibliographie}

ANDERSON (L.J.) and SEVEL (T.) - 6 years Environmental Tritium Profiles in the Unsaturated and Saturated zones. Gronhoj, Denmark. Symposium Isotopes Techniques in Groundwater Hydrology. I.A.E.A., Vienne, SM 182/1. 1974.

BEAR (J.) -- Dynamics of fluids in porous media. American Elsevier Publ. Co., New-York, 764 pp. 1972.

BIGGAR (J.W.) NIELSEN (D.R.) - Miscible Displacement and Leaching Phenomena. Irrigation of Agricultural Lands, Agron, Monogr. $\mathrm{n}^{\circ}$ 11, p. 254-274. 1967

BRENNER (H.) - Longitudinal Diffusion in Packed Beds of Finite Length. Numerical values. Chem. Eng. Sci. Vol. 17, 229-243, 1962.

CHAUDHARI (N.M.) - An improved Numerical Technique for Solving Multidimensional Miscible Displacement Equations. Soc. of Petr. J. Vol. 11, p. 277-284. 1971 .

CJATS (K.H.), SMITH (B.D.) - Dead End pore volume and Dispersion n Porous Media. Soc. of Petr. Eng. J. - Vol. 4, p. 73-84. 1964

DAGAN (G.) - Some Theoretical Aspects of Vertical Miscible Displacements with Density Gradients in Unsaturated Porous Media. Research Sponsored by A.E.C. project $n^{\circ}$ 401-25-07.1966.

Fried (J.J.), Combarnous (M.A.) - Dispersion in Porous Media. Advances in Hydroscience, Vol. 6, p. 142-249. 1971.

GAUDET (J.P.) - Ecoulement transitoire d'eau et de sel dans la zone non saturée. Application à l'étude de la contamination des nappes à surface libre. Thèse de 3ème cycle. Université Scientifique et Médicale de Grenoble. 1974.

JEGAT (H.) - Etude expérimentale et simulation d'écoulement de fluides miscibles en milieu poreux non saturé. Thèse de DocteurIngénieur, Université Scientifique et Médicale de Grenoble. 1975.

KLOTZ (D.) MOSER (H.) - Hydrodynamic Dispersion as Aquifer Characteristic Model Experiments by Means of Radioactive Tracers. Symp. on Isotope Techniques in Ground Water Hydrology. IAEA. Vienne SM 182/42. 1974

LAPIDUS (L.) and AMUNDSON (N.R.) - Mathematics of adsorption in beds. VI The effect of longitudinal diffusion in exchange and chromatographic columns J. Phys. Chem. 56:984-988. 1952.

PFANNKUCH (H.O.) - Contribution à l'étude des déplacements de fluides miscibles dans un milieu poreux. Thèse de Docteur-Ingénieur - Université de Paris. 1962.

VACHAUD (G.), THONY (J.L.) - Hysteresis effects during infiltration and redistribution in a soil column at different water contents. Water Resources Res. 7 : 111-127. 1970.

VAN Genuchten (M.T.) - Mass Transfer Studies in Sorbing Porous Media. Ph. D. Thesis - New-Mexico State University. 1974.

VAN GENUCHTEN (M.T.), DAVIDSON (J.M) and WIERENGA (P.J.) - An evaluation of kinetic and equilibrium equations for the prediction of pesticide movement through porous media. Soil Sci. Soc. Amer. Proc. 38 : 29-35. 1974.

VILLERMAUX (J.) and VAN SWAAY (W.P.M.) - Modèle représentatif de la distribution des temps de séjour dans un réacteur semi in fini à dispersion axiale avec zones stagnantes. Chem. Eng. Sci. $24: 1097-1111: 1969$. 


\section{Discussion}

Président : M. Jean MARGAT

M. Le Président remercie les Conférenciers pour leur intéressant exposé et ouvre la discussion.

"Vous avez montré, dit M. PEA UDECERF (B.R.G.M.), que le modèle à trois paramètres permet de retrouver avec une bonne approximation les courbes relevées au cours de vos expériences. Vous attribuez cette conformité entre le calcul et l'observation au fait que le modèle tient compte des zones d'eau morte ; mais cela ne provient-il pas, en grande partie, du fait que vous disposez - pour l'ajustement de votre modèlede trois paramètres au lieu d'un seul dans les modèles "convectifsdispersifs"?

\section{GAUDET répond :}

"Nous avons fait une optimisation des paramètres par une technique de "pattern search" qui nous permet de dire que si l'on ne peut prouver rigoureusement l'unicité des solutions, on ne peut non plus trouver deux solutions satisfaisantes avec deux trio de paramètres",

M. de MARSILY (Ecole des Mines, Fontainebleau), suggère une autre solution :

"Ne peut-on interpréter, dit-il, votre terme d'échange "phase mobile phase immobile":

$$
\theta m \frac{\delta C_{i m}}{\delta t}=\alpha\left(C_{m}-C_{i m}\right)
$$

comme une diffusion, par agitation moléculaire, des molécules tracées de la phase mobile dans l'eau de la phase immobile?

"Dans ce cas, le paramètre $\alpha$, en passant d'une substance à une autre, doit pouvoir être relié au coefficient de diffusion moléculaire de la substance dans l'eau.

"Ayant mesuré $\alpha_{1}$ avec une expérience pour une substance 1 , ne pourrait-on pas alors prévoir $\alpha_{2}$, pour une substance 2 , et vêrifier expérimentalement la validité de $\alpha_{2}$. Cette approche permettait de confirmer la réalité physique du paramètre $\alpha$.

M. GUIZERIX intervient en ces termes:

"Les interventions précédentes appelient les commentaires suivants :

"En premier lieu, on a toujours intérêt, et cela devient un lieu commun de le souligner, à regarder ce qui se fait dans les disciplines voisines. Ainsi pendant longtemps, les spécialistes des traceurs en hydrologie ont ignoré ce qui se faisait dans le domaine de la physiologie et cela était très regrettable car les méthodes de traceur étaient plus avancées dans ce dernier domaine.

"Le modèle à trois paramètres dont on vient de discuter, aujourd'hui, à propos d'écoulements dans la zone saturée, a été développé, si ma mémoire est bonne en 1969, par Villermaux à Nancy pour les colonnes à garnissage, utilisées dans le génie chimique. Il était depuis longtemps prévisible qu'il serait appliqué en hydrológie.

"A son propos, je ne suis pas tout à fait d'accord avec l'assertion de M. PEA UDECERF, qui nous dit qu'avec trois paramètres on décrit n'importe quoi ou tout au moins beaucoup de choses; cela est vrai en partie mais dans le cas présent, les trois paramètres ont un fondement physique, dispersion, volumes relatifs et taux de transfert, et il n'est donc pas étonnant que ce modèle habille au mieux la réalité".

Pour M. Fontes, dans la réalité, le découpage entre les zones d'eau mobile et les zones d'eau immobile est arbitraire ; il faudrait étudier plus à fond la structure de la perméabilité car la dispersivité dépend étroitement de la dispersion des perméabilités.

Revenant aux essais décrits par M. GAUDET, M. FONTES critique l'emploi dans ces derniers d'une solution de chlorure de calcium. En présence de bicarbonate avec un $\mathrm{pH}$ voisin de la neutralité, elle peut donner lieu à un précipité de calcite.
Les dosages en chorures et en calcium exécutés sur l'effluent n'ont montré aucune différence de cinétique, ce qui montre que la précipitation signalée par M. FONTES ne s'est pas produite au cours de nos expériences, rétorque $\mathrm{M}$. JEGAT.

Pour M. BONNET, le malentendu -s'il existe- réside dans un problème d'échelle; dans les expériences de laboratoire, on élimine la convection en travaillant sur des "colonnes" homogènes avec des vitesses de percolation uniformes ; sur le terrain, au contraire, c'est le problème de la répartition des vitesses qui intéresse, en priorité, le praticien.

Mais, observe M. GAUDET, nos travaux intéressent essentiellement des zones de faible dimensions (de l'ordre du mètre) et le traitement mathématique que nous proposons ne saurait ètre étendu à l'échelle des nappes souterraines. Toutefois, des problèmes tels que : l'efficacité des engrais dans la zone racinaire, le lessivage d'un sol, sont directement concernés par notre étude.

En s'aidant de la projection de quelques photographies de tranches de terrains naturels tirées d'une étude récente (1), M. MO LIN A R I montre l'hétérogénéité de la circulation de l'eau dans les nappes alluviales comportant des stratifications entrecroisées, des lentilles poreuses au sein de matériaux quasi-imperméables, etc...

"Vous voyez, conclut M. Molinari, la discordance considérable entre les schémas que l'on peut réaliser au laboratoire et la réalité. Nous nous attachons néamoins à approcher cette réalité par un modèle, certes sommaire, mais un peu plus proche du réel que celui que l'on a l'habitude d'utiliser".

M. Paul Ch. LEVEQue félicite M. Molinari de son souci de réalisme et poursuit :

"Une couche d'alluvions perméables étudiée sur une hauteur de 20 ou $30 \mathrm{~m}$ ne comprend, en fait, que peu de terrain réellement perméable C'est ce que M. MOLINARI vient de nous montrer et il a parfaitement raison. Toutefois, les géologues sont déjà au courant de cette répartition.

"Nous avons tenté de concrétiser ces faits depuis assez longtemps, avec les traceurs. Grâce à leur emploi, nous retrouvons, aujourd'hui, cette localisation. Nous avons aussi essayé, et peut être à moitié réussi, de matérialiser cette hétérogénéité par une photographie des zones perméables. Nous avons retrouvé toutes les données nécessaires à la "localisation" des passages perméables tant en zone saturée qu'en zone non saturée.

"Tout ceci montre bien que les difficultés dont parlait M. BONNET tout à l'heure ne seront levées qu'en tenant compte des couches vraiment perméables. Ainsi, sur une série alluviale, il faudrait considérer seulement $10 \%, 5 \%$-ou même moins- de la couche réellement perméable. Mème si le reste est poreux et a une perméabilité élevée en laboratoire, l'eau dans la nature refuse d'y passer parce qu'il existe quelque part une limite imperméable.

"C'est pourquoi, m'adressant aux théoriciens qui ont étudié ces problèmes de façon remarquable, je crois qu'il faudrait travailler à l'échelle dont vous parliez tout à l'heure, c'est-à-dire celle du décimètre ou au maximum du mètre. Lorsqu'on saura ce qui se passe à cette échelle, on aura résolu $80 \%$ des problèmes des circulations à l'intérieur des alluvions saturés ou non",

Sur ces sages paroles, dit M. Le Président, je vais clore la discussion et donner la parole à M. MARCE pour l'exposé de la communication qu'il a préparée en collaboration avec MM. CASTANY et MARGAT.

(1) J. MOlinari, P. CALMELS, J.F. BUSSE : Propagation de la pollution entre le sol et la nappe (zone non saturée) dans la plaine de l'Est lyonnais. Revue de l'Institut Pasteur de Lyon 1974, t. $7, n^{\circ} 4$ (p. 347 à 371). 


\section{Abstract \\ Transfer of solutes in unsaturated soil in presence of stagnant water}

Assuming that when a soil volume is unsaturated part of the water can be trapped by air between particles and in dead end pores, the classical scheme of hydrodynamic dispersion, represented by eq. (1), is modified in order to be more representative with the criterion that during the movement of solutes in soil lateral diffusion of chemicals in or out of the stagnant water is possible. The change of salt concentration during a steady flow is now represented by the set of equations (2) and (3) where subscripts $m$ and $i m$ refers to the mobile and immobile fraction respectively. Three parameters must be known in order to solve this set of equations : the apparent dispersion coefficient $D\left(\mathrm{~cm}^{2} / \mathrm{hr}\right)$; the diffusion mass transfer coefficient $\alpha\left(\mathrm{hr}^{-1}\right)$ and the mobile fraction $f=\theta_{m} / \theta$.

A series of experiments where done in a vertical soil column of soil for steady state flow of water and a pulse or step change of salt concentration at the inlet. The salt concentration where measured with time at several depths inside and at the exit of the column. Values for $D_{a p}, \alpha, f$ were obtained by curve fitting observed and calculated concentration, at one depth $(22 \mathrm{~cm})$ using an explicit discretization of eq. (2). The same values, first determined during a step change of salt concentration, corresponding to the experiment on fig. 3 , were first used to calculate salt distribution at other depths and at the exit for this experiment, and then to compute salt distribution during an other experiment, done with the same water flux, but for a pulse change of salt concentration (fig. 4). Excellent agreement was obtained in both case ; the model presented a good description of the extensive tailing observed inside and at the outside of the column, which could not be simulated with the classical "dispersion-convective" equation (fig. 3.a). Finally a sensitivity study of the influence of each parameters was done (fig. 5 to 7 ). It appears that the immobile fraction of water has the strongest influence on the relative concentration curves.

Further experiments should now be done in order to determine the variations of each parameter with the water content and to solve the transient infiltration problems. 